\title{
Effect of Bitter Melon on Spatial Memory of Rats Receiving a High-Fat Diet
}

This article was published in the following Dove Press journal:

Journal of Experimental Pharmacology

\section{Hamid Sepehri' \\ Arefeh Hojati $^{2}$ \\ Razieh Safari ${ }^{3}$}

'Neuroscience Research Center, Golestan University of Medical Sciences, Gorgan, Iran; ${ }^{2}$ Department of Physiology, Faculty of Medicine, Golestan University of Medical Sciences, Gorgan, Iran; ${ }^{3}$ Medical Cellular and Molecular Research Center, Golestan University of Medical Sciences, Gorgan, Iran
Correspondence: Hamid Sepehri Neuroscience Research Center, Golestan University of Medical Sciences, Gorgan, Iran

Email Hamid.Sepehri@yandex.com

\begin{abstract}
Introduction: Momordica charantia or bitter melon is a tropical vine of the family Cucurbitaceous widely grown in India. Its fruits have potent anti-oxidant properties due to the presence of tannins, vitamin $\mathrm{C}$ and flavonoids. There is much evidence it protects cognitive function and cholesterol level. In addition, there are reports of the effect of a high-fat diet (HFD)on memory. In this study, the effect of bitter melon on spatial memory in rats, following an HFD, in a water maze was examined.

Material and methods: In this study, 28 male Wistar rats aged 10 weeks and weighing between 180 and 250 grams were divided into four groups $(\mathrm{N}=7)$. Control, High-fat, High-fat + bitter melon, and bitter melon. The control group was fed a standard rat diet, whereas the high-fat groups were fed the same standard diet containing $5 \%$ cholesterol for eight weeks, and the treated group received, in addition, $1 \mathrm{~g} / \mathrm{kg}$ bitter melon fruit powder in their diet, Learning and spatial memory were evaluated by using a Morris Water Maze (MWM) for a six-day period, including five days of training, the last day was the test day (probe day).

Results: The high-fat group was fed a high-fat diet for two months, this resulted in reduced learning ability;, this group took longer and travelled a longer distance compared to the control group. However, the administration of bitter melon improved memory function only in the high-fat group.
\end{abstract}

Conclusion: The administration of bitter melon improves spatial-memory performance in rats receiving an HFD.

Keywords: bitter melon, high-fat diet, water maze

\section{Introduction}

In recent decades, the increase of high-fat diets (HFD) has become a worldwide health problem. Excessive dietary intakes of fats negatively affect blood parameters, including plasma lipids, cholesterol, and triglycerides. Hyperlipidemia-induced brain function deficiencies, cognitive impairments, and behavioural changes have been studied extensively in HFDs. ${ }^{1,2}$ The negative effects that hyperlipidemia and obesity have on human health have prompted the search for effective treatments and interventions. Pharmaceutical drug interventions have considerable side effects. Therefore, Complementary and alternative medicine involves the use of herbs and other dietary supplements as alternatives to pharmaceutical drugs. Momordica charantia (M. charantia) has received attention for its anti-hyperlipidemia properties. ${ }^{3}$ M. charantia, also known as bitter melon, karela, is a popular plant used throughout Asia and India. ${ }^{4}$ Various medicinal properties such as antidiabetic, anthelmintic, antimalarial, antibacterial, antiviral and anticancer properties are claimed for bitter melon. ${ }^{5}$ Several experiments have shown the hypo-cholesterolemic effects of bitter 
melon. ${ }^{3,6,7}$ Feeding bitter melon seeds to soil-fed rats significantly decreased the plasma lipid peroxidationand increased phospholipid concentrations in heart and brain. ${ }^{8}$ Recently, some reports have shown neuroprotective effects of bitter melon against cerebral ischemia-reperfusion induced neuronal injury and consequent neurological deficits in diabetic mice. ${ }^{9}$ In other studies, antiamnesic activity, through inhibiting lipid peroxidation and decreasing acetyl cholinesterase activity in the brain, has been reported. ${ }^{10}$ Thus, a combination of antioxidant, anti-inflammatory and cholesterol-lowering properties exhibited by bitter melon lead us to investigate the effects of bitter melon on cognations in rats in a Morris Water Maze (MWM) who had been fed a high-fat diet.

\section{Materials and Methods}

\section{Animals}

In this study, 28 male Wistar rats aged 10 weeks and weighing between 180 and 250 grams were divided into four groups $(\mathrm{N}=7)$. A control group $(\mathrm{C})$, a high-fat diet group (HFD) and a HFD + bitter melon group (HB), and a control + bitter melon (CB). The Control group had ad libitum access to a standard rat diet for 30 days. The study was performed according to the guidelines for laboratory animal use and care set by the Animal Ethics Committee of Golestan University of Medical Sciences (GOUMS), with ethical code 970426073. The CB group was fed with that same standard rat food which contains $1 \mathrm{~g} / \mathrm{kg}$ bitter melon fruit powder. The C and CB groups had ad libitum access to the same diet, which also contained 5\% cholesterol (SigmaAldrich, Steinheim, Germany) and 5\% cholesterol $+1 \mathrm{~g} / \mathrm{kg}$ bitter melon fruit powder respectively, for 30 days.

\section{Morris Water Maze Test}

In this study, the MWM method was used for spatial memory. The MWM is a circular tank with black walls (140 cm diameter and $60 \mathrm{~cm}$ depth) filled with water up to $30 \mathrm{~cm} .{ }^{11}$ The maze was supposedly divided into four quarters - North, South, East and West. A rescue platform was in one of these quarters, positioned on the bottom of the tank with a metal stand, $1.5 \mathrm{~cm}$ below the surface of water (out of the rats' sight). The animals received five days training with the hidden platform, each day included four training sessions with a $60 \mathrm{~s}$ intersession interval. Each trial began by placing a rat at one of the three starts points, with its face toward the wall of the pool. The start location varied on each training trial and changed each day. The trial was terminated when the animal entered the platform. If the rat did not find the platform within 60 seconds it was placed on the platform by the experimenter for 15 seconds. During acquisition of the spatial navigation task all groups were given one session of four trials each day (day 1-5; trial 1-20). Spatial memory was evaluated in the probe trial, on the sixth day (trial 21). The platform was removed and animals were allowed to swim for 60 seconds. The path of the animals in the maze was monitored using a computerized video tracking system (Maze router, urmia Instruments Inc). The parameters measured were the time taken to reach the platform (latency) and swimming speed, swim path length (SPL) in the training trials. And during probe trials was the percentage run time within the quadrant of the water bath where the hidden platform had been placed in the training trials.

\section{Ethical Considerations}

The study is approved by ethical committee of Golestan University of Medical Sciences.

\section{Statistics Analysis}

All the experimental results were cantered using seven mice in each group with Maze router software. Escape latency values were evaluated by two-way repeatedmeasures ANOVA. Experimental results were expressed as the mean \pm SEM. Differences between data sets were considered significant at $\mathrm{P}$ less than 0.05 .

\section{Results}

The effects of an HFD and bitter melon treatment on weight and plasma lipid parameter are shown in Table 1. The body weight in the HFD group was significantly more than other

Table I Effect of Experimental Diet on Body Weight and Cholesterol Profile

\begin{tabular}{|c|c|c|c|c|}
\hline & Control & High-Fat & $\begin{array}{l}\text { High-Fat + } \\
\text { Bitter } \\
\text { Melon }\end{array}$ & $\begin{array}{l}\text { Bitter } \\
\text { Melon }\end{array}$ \\
\hline $\begin{array}{l}\text { Total } \\
\text { cholesterol }\end{array}$ & $60 \pm 11$ & $140 \pm 25 * * *$ & $70 \pm 8$ & $62 \pm 15$ \\
\hline LDL (mg/dl) & $6 \pm 1.3$ & $12 \pm 2.5 * *$ & $6 \pm 2.5$ & $5.4 \pm 0.4$ \\
\hline $\begin{array}{l}\text { Triglyceride } \\
(\mathrm{mg} / \mathrm{dl})\end{array}$ & $58.6 \pm 11$ & $84 \pm 9 * *$ & $60 \pm 8$ & $55 \pm 5$ \\
\hline Body weight (g) & $220 \pm 7$ & $245 \pm 13 *$ & $235 \pm 8$ & $224 \pm 5$ \\
\hline
\end{tabular}

Notes: Means \pm SE of seven rats significantly differed from the control group. $*(\mathrm{p}<0.05) * *(\mathrm{p}<0.01) * * *(\mathrm{P}<0.00 \mathrm{I})$. 
groups at the end of the experimental period $(\mathrm{P}<0.05)$ Treatment of the HFD group with bitter melon decreased body weight to near the control-group weight, with no significant difference, but treatment with bitter melon with the standard diet did not change body weight and no significant differences were observed when compared with the control group.

Similarly, the blood-serum Total Cholesterol (TC), Total Glyceride (TG), Low-density lipoprotein (LDL) levels were higher in the HFD comparison with the other groups $(\mathrm{P}<0.01)$. Bitter melon treatment significantly reduced $\mathrm{TC}$, TG, LDL levels in the HFD group to the level observed in animals fed the standard diet with no significant difference. But no significant deference was observed in the-bitter melon group compared to the control group.

\section{The Effect of a High-Fat Diet and Bitter Melon Treatment on the Morris Water Maze Test}

A two-way repeated-measures ANOVA showed a significant group day interaction $\mathrm{P}<0.01$ for escape latency in the MWM test (Figure 1). The statical analysis indicated that control animals showed a decrease in escape latency over the five-day testing period, indicating that they successfully learned the location of the platform; however, in animals fed the HFD, the escape latency did not show a significant change during the test period and on day five and it was significantly $(\mathrm{P}<0.01)$ higher than the control group. After the administration of bitter melon, a significant difference was observed when compared to the high-fat group during all experiment days $(\mathrm{P}<0.01)$, indicating memory improvement. However, no significant differences were observed between the control group and rats which were only treated with bitter melon $(\mathrm{P}>0.01)$. After the learning steps in the MWM, memory retrieval was evaluated by removing the hidden platform from the maze. At this stage, the time spent

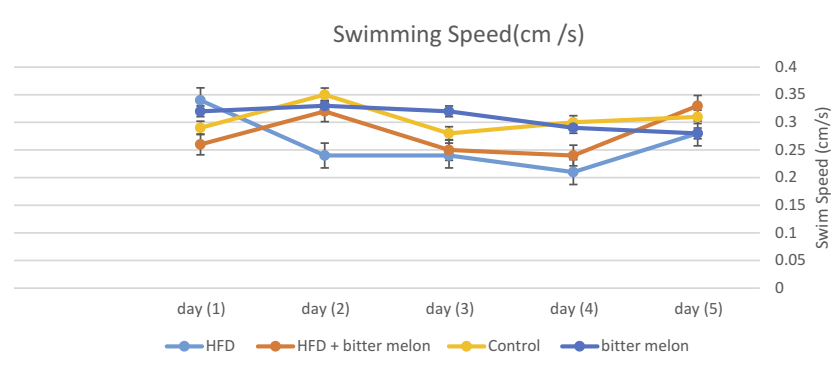

Figure I Effect of a high-fat diet (HFD) and bitter melon treatment on the performance of spatial-memory acquisition phase in a Morris Water Maze. Swimming speed of each day (made up of four trials). in the location of the platform was evaluated. Results showed that the group with the HFD spent less time in the targeted zone, which differed significantly with the control group $(\mathrm{P}<0.05)$. However, the groups treated with bitter melon spent more time in the targeted zone, which also had a significant difference with the results of the group with the HFD $(\mathrm{P}<0.05)$. This indicates the positive impact of bitter melon on the spatial memory of rats. The time spent in the target zone in the bitter melon group did not show significant differences with the control group (Figure 2). The analysis of swimming speed by using a two-way ANOVA also showed no significant differences among the groups as the training days progressed, and no interaction between days and groups (Figure 3).

\section{Discussion}

On day 60 the plasma LDL-cholesterol and triglyceride in the HFD group were higher than that control group, which is in accordance with other studies. ${ }^{12,13}$ Treatment of HFD

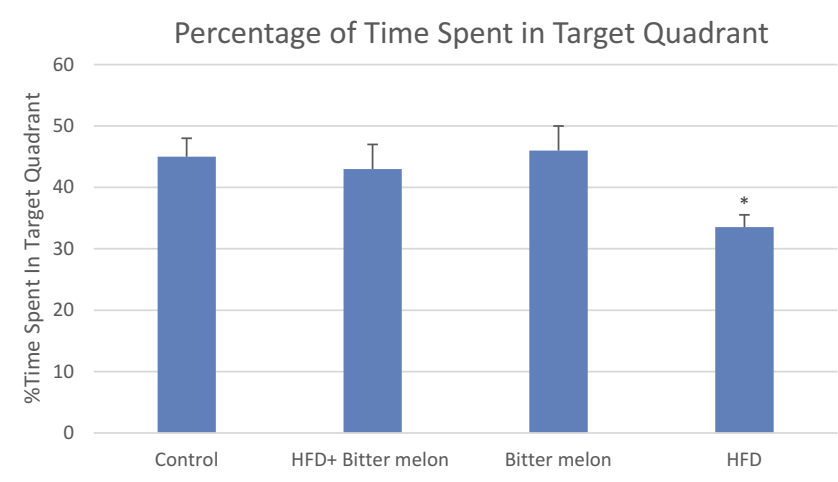

Figure 2 Water maze mean $( \pm S E M)$ percentage of time spent in target quadrant in a Morris Water Maze during probe trials. Asterisks indicate a significant difference from control $\left({ }^{*} \mathrm{p}<0.05\right)$.

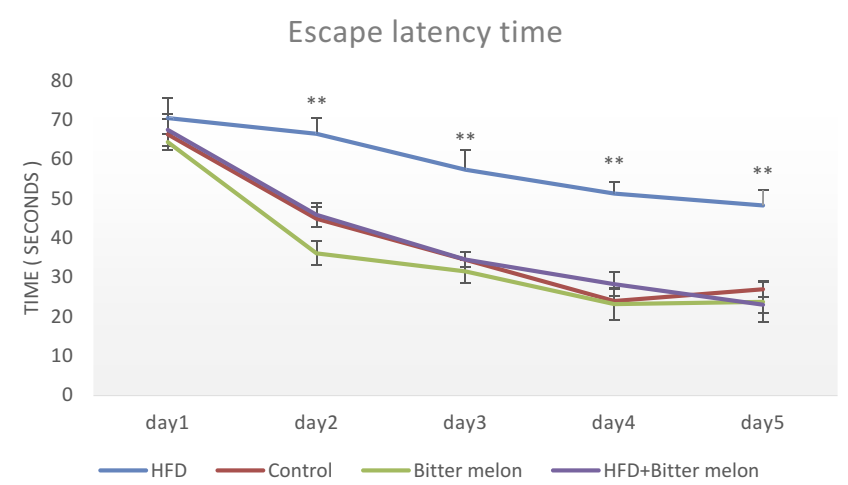

Figure 3 Effect of a high-fat diet (HFD) and bitter melon treatment on the performance of spatial-memory acquisition phase in a Morris Water Maze. Average escape latency within each day (made up of four trials). Asterisks indicate a significant difference from control (**p $<0.01$ ). 
rats with bitter melon decreased plasma lipids nearer to the control group. There were no significant differences in LDL- between the bitter melon and the control groups. Similar results have been reported in other studies. ${ }^{7,14,15}$ In this study, rats were trained for five days and behavior testing was carried out on day six. The results showed a significant reverse correlation between the HFD and the learning ability of rats in the spatial memory. The absence of alterations in spontaneous locomotor activity indicates that the results of the cognitive and behavioral tests were not affected by changes in the animals' motor activity. Treatment with the HFD for two months led to poor spatial-memory performance in rats. Several studies have been performed on the relationship of learning when fed HFD. ${ }^{16,17}$ It could be related to oxidative stress, because diet-induced obesity can increase free radical generation and oxidative stress in brains of rodents. ${ }^{18,19}$ It has been widely reported that intense oxidative stress is one of the main causal factors involved in the impairment of cognition. $^{20,21}$ Previous studies showed that bitter melon seeds possess potent antioxidant properties, which may be directly or indirectly responsible for its cognitive enhancement effects. ${ }^{22}$ The effect of bitter melon on HFD-fed animals might be directly related to their lipid-lowering properties. Recently, several reports have shown a strong link between high cholesterol levels and incidence of Alzheimer's disease. ${ }^{23}$ Clinical studies suggested that the brain's cholesterol concentration is regulated by serum cholesterol level and that there is a relationship between the brain and peripheral cholesterol. ${ }^{24}$ Clinical studies suggested that the net brain cholesterol concentration is regulated by serum cholesterol level and that there is a link between the central nervous system and peripheral cholesterol pools. It has been reported that chronic oral administration of ethanolic extract of bitter melon significantly decreased lipid levels, such as cholesterol and triacylglyceride (TAG), in serum and liver in rats. Hypercholesterolemia in rats impairs the cholinergic system and leads to memory deficits. ${ }^{25}$ In addition, changes in lipid profiles in other mechanisms are likely to be responsible for the actions of bitter melon on cognitive function. In animals fed the standard diet, the cognitive parameters did not significantly improve following bitter melon treatment with no changes in spatial tasks.

Furthermore, animal studies have documented that experimental obesity can be associated with neural changes $^{26}$ and in the expression of synaptic marker proteins. $^{27,28}$ Thus, the cognitive decline mentioned in this study associated with HFD rats might be secondary to structural alterations induced by HFD. Another possibility could be related to the insulin-like effect of bitter melon, increased absorption of insulin can improve cognitive tasks. ${ }^{6}$ Recent studies have shown that insulin, in addition to the regulation of energy supply, is required for optimal hippocampal memory processing. ${ }^{29}$

\section{Conclusion}

It can be concluded that the eight-week HFD reduces the learning ability of rats in a spatial task, such as the MWM. M. charantia lowered the serum cholesterol and triglyceride in rats, and, ultimately, improved the spatial memory of rats after it was administered for eight weeks.

\section{Acknowledgments}

This article has been derived from an MD thesis financially supported by the Golestan University of Medical Sciences.

\section{Disclosure}

The authors report no conflicts of interest in this work.

\section{References}

1. Freeman LR, Haley-Zitlin V, Rosenberger DS, Granholm A-C. Damaging effects of a high-fat diet to the brain and cognition: a review of proposed mechanisms. Nutr Neurosci. 2014;17 (6):241-251. doi:10.1179/1476830513Y.0000000092.

2. Granholm A-C, Bimonte-Nelson HA, Moore AB, Nelson ME, Freeman LR, Sambamurti K. Effects of a saturated fat and high cholesterol diet on memory and hippocampal morphology in the middle-aged rat. J Alzheimers Dis JAD. 2008;14(2):133-145. doi:10. 3233/JAD-2008-14202

3. Ahmed I, Lakhani MS, Gillett M, John A, Raza H. Hypotriglyceridemic and hypocholesterolemic effects of anti-diabetic momordica charantia (karela) fruit extract in streptozotocin-induced diabetic rats. Diabetes Res Clin Pract. 2001;51(3):155-161. doi:10. 1016/S0168-8227(00)00224-2

4. Cefalu WT, Ye J, Wang ZQ. Efficacy of dietary supplementation with botanicals on carbohydrate metabolism in humans. Endocr Metab Immune Disord Drug Targets. 2008;8(2):78-81. doi:10.2174/1871 53008784534376

5. Grover JK, Yadav SP. Pharmacological actions and potential uses of momordica charantia: a review. $J$ Ethnopharmacol. 2004;93 (1):123-132. doi:10.1016/j.jep.2004.03.035.

6. Jayasooriya AP, Sakono M, Yukizaki C, Kawano M, Yamamoto K, Fukuda N. Effects of momordica charantia powder on serum glucose levels and various lipid parameters in rats fed with cholesterol-free and cholesterol-enriched diets. J Ethnopharmacol. 2000;72(1-2):331-336. doi:10.1016/S0378-8741(00)00259-2

7. Noguchi R, Yasui Y, Suzuki R, Hosokawa M, Fukunaga K, Miyashita K. Dietary effects of bitter gourd oil on blood and liver lipids of rats. Arch Biochem Biophys. 2001;396(2):207-212. doi:10. 1006/abbi.2001.2624.

8. Dhar P, Bhattacharyya DK. Nutritional characteristics of oil containing conjugated octadecatrienoic fatty acid. Ann Nutr Metab. 1998;42 (5):290-296. doi:10.1159/000012746. 
9. Malik ZA, Singh M, Sharma PL. Neuroprotective effect of momordica charantia in global cerebral ischemia and reperfusion induced neuronal damage in diabetic mice. J Ethnopharmacol. 2011;133 (2):729-734. doi:10.1016/j.jep.2010.10.061.

10. Pathakota R. Neuroprotective effects of momordica charantia on scopolamine induced alzheimer's disease. World J Pharm Pharm Sci. 2017;2141-2155. doi:10.20959/wjpps201704-9025.

11. Vorhees CV, Williams MT. Morris water maze: procedures for assessing spatial and related forms of learning and memory. Nat Protoc. 2006;1(2):848-858. doi:10.1038/nprot.2006.116.

12. Cicero AFG, Vitale G, Savino G, Arletti R. Panax notoginseng (Burk.) effects on fibrinogen and lipid plasma level in rats fed on a high-fat diet. Phytother Res. 2003;17(2):174-178. doi:10.1002/ptr.1262.

13. Yun SN, Ko SK, Lee KH, Chung SH. Vinegar-processed ginseng radix improves metabolic syndrome induced by a high fat diet in ICR mice. Arch Pharm Res. 2007;30(5):587-595. doi:10.1007/BF02977653

14. Kinoshita H, Ogata Y Effect of bitter melon extracts on lipid levels in Japanese subjects: a randomized controlled study. Available from: https:// www.hindawi.com/journals/ecam/2018/4915784/. Accessed September 13, 2019.

15. Alam MA, Uddin R, Subhan N, Rahman MM, Jain P, Reza HM. Beneficial role of bitter melon supplementation in obesity and related complications in metabolic syndrome. J Lipids. 2015;2015:1-18. doi: $10.1155 / 2015 / 496169$.

16. Winocur G, Greenwood CE. The effects of high fat diets and environmental influences on cognitive performance in rats. Behav Brain Res. 1999;101(2):153-161. doi:10.1016/S0166-4328(98)00147-8

17. Cordner ZA, Tamashiro KLK. Effects of high-fat diet exposure on learning \& memory. Physiol Behav. 2015;152(Pt B):363-371. doi:10. 1016/j.physbeh.2015.06.008.

18. Zhang X, Dong F, Ren J, Driscoll MJ, Culver B. High dietary fat induces NADPH oxidase-associated oxidative stress and inflammation in rat cerebral cortex. Exp Neurol. 2005;191(2):318-325. doi:10.1016/j.expneurol.2004.10.011.

19. Souza CG, Moreira JD, Siqueira IR, et al. Highly palatable diet consumption increases protein oxidation in rat frontal cortex and anxiety-like behavior. Life Sci. 2007;81(3):198-203. doi:10.1016/j. lfs.2007.05.001.
20. Calabrese V, Butterfield DA, Stella AMG. Nutritional antioxidants and the heme oxygenase pathway of stress tolerance: novel targets for neuroprotection in alzheimer's disease. Ital J Biochem. 2003;52 (4):177-181

21. White CL, Pistell PJ, Purpera MN, et al. Effects of high fat diet on morris maze performance, oxidative stress, and inflammation in rats: contributions of maternal diet. Neurobiol Dis. 2009;35(1):3-13. doi:10.1016/j.nbd.2009.04.002.

22. Sathishsekar D, Subramanian S. Antioxidant properties of momordica charantia (bitter gourd) seeds on streptozotocin induced diabetic rats. Asia Pac J Clin Nutr. 2005;14(2):153-158.

23. Sjögren M, Blennow K. The link between cholesterol and alzheimer's disease. World J Biol Psychiatry off J World Fed Soc Biol Psychiatry. 2005;6(2):85-97. doi:10.1080/15622970510029795

24. Orth M, Bellosta S. Cholesterol: its regulation and role in central nervous system disorders. Cholesterol. 2012;2012:1-19. doi:10.1155/ $2012 / 292598$.

25. Ullrich C, Pirchl M, Humpel C. Hypercholesterolemia in rats impairs the cholinergic system and leads to memory deficits. Mol Cell Neurosci. 2010;45(4):408-417. doi:10.1016/j.mcn.2010.08.001.

26. Stranahan AM, Norman ED, Lee K, et al. Diet-induced insulin resistance impairs hippocampal synaptic plasticity and cognition in middle-aged rats. Hippocampus. 2008;18(11):1085-1088. doi:10.10 02/hipo. 20470 .

27. Molteni R, Barnard RJ, Ying Z, Roberts CK, Gómez-Pinilla F, HighFat A. Refined sugar diet reduces hippocampal brain-derived neurotrophic factor, neuronal plasticity, and learning. Neuroscience. 2002;112(4):803-814. doi:10.1016/s0306-4522(02)00123-9.

28. Wu A, Ying Z, Gomez-Pinilla F. The interplay between oxidative stress and brain-derived neurotrophic factor modulates the outcome of a saturated fat diet on synaptic plasticity and cognition. Eur J Neurosci. 2004;19(7):1699-1707. doi:10.1111/j.1460-9568.2004. 03246.x.

29. McNay EC, Ong CT, McCrimmon RJ, Cresswell J, Bogan JS, Sherwin RS. Hippocampal memory processes are modulated by insulin and high-fat-induced insulin resistance. Neurobiol Learn Mem. 2010;93(4):546-553. doi:10.1016/j.nlm.2010.02.002.

\section{Publish your work in this journal}

The Journal of Experimental Pharmacology is an international, peerreviewed, open access journal publishing original research, reports, reviews and commentaries on all areas of laboratory and experimental pharmacology. The manuscript management system is completely online and includes a very quick and fair peer-review system. Visit http://www.dovepress.com/testimonials.php to read real quotes from published authors. 